\title{
A mini outbreak of human metapneumovirus infection with severe acute respiratory symptoms in a selected group of children presented to a teaching hospital in Sri Lanka
}

\author{
Faseeha Noordeen $^{1}\left(\mathbb{D} \cdot\right.$ F. N. Nagoor Pitchai $^{1} \cdot$ S. Thushara Kudagammana ${ }^{2,3} \cdot$ \\ R. A. Mohamed Rafeek ${ }^{1}$
}

Received: 7 February 2019/Accepted: 14 March 2019/Published online: 4 April 2019

(C) Indian Virological Society 2019

\begin{abstract}
Human metapneumovirus (hMPV) of the family Paramyxoviridae is a relatively new virus causing severe acute respiratory tract infections (SARI) in children. Data on hMPV infection in Asia including Sri Lanka is limited. We aimed to detect respiratory viruses including hMPV in a selected group of children affected by a small outbreak of SARI presented to the Teaching Hospital, Peradeniya (THP), Sri Lanka in 2014. Nasopharyngeal aspirates (NPA) were obtained from 21 children with SARI and tested for hMPV, influenza A and B, parainfluenza 1, 2 and 3 (PIV $1-3$ ), adenovirus and respiratory syncytial virus (RSV) antigens using an immunofluorescence assay (IFA). In addition, a one step RT-PCR was done for the detection of hMPV from the viral RNA extracts. Of the 21 NPA samples tested for respiratory viral antigens by IFA, two were positive for RSV (9.5\%), one was positive for influenza A (4.8\%) and one was positive for both adenovirus and PIV-2 (4.8\%). Of the 21 NPA viral RNA extracts tested by RTPCR, $18(86 \%)$ were positive for hMPV, in which 2 were co-infected with RSV and influenza A virus, respectively. hMPV was the predominant cause of SARI outbreak (2014) in children presented to the THP, Sri Lanka.
\end{abstract}

Keywords Human metapneumovirus - Severe acute respiratory infections · Children · IFA · RT-PCR

Faseeha Noordeen

faseeha.noordeen12@gmail.com; faseehan@pdn.ac.lk

1 Department of Microbiology, Faculty of Medicine, University of Peradeniya, Peradeniya, Sri Lanka

2 Department of Paediatrics, Faculty of Medicine, University of Peradeniya, Peradeniya, Sri Lanka

3 Teaching Hospital, Peradeniya, Sri Lanka
Acute respiratory tract infections (ARTI) including severe acute respiratory tract infections (SARI) are common in children. The mortality rate in SARI is high in developing countries compared to developed countries although the morbidity rate remains more or less the same globally [1]. According to the global burden of diseases (GBD) study in 2015, ARTIs including SARI caused 2.74 million deaths in children under 5 years of age [2]. Around $70 \%$ of these deaths have been reported from Africa and Southeast Asia [3]. ARTIs in children are commonly caused by viruses such as influenza $\mathrm{A}$ and $\mathrm{B}$, respiratory syncytial virus (RSV), parainfluenza virus 1,2 and 3 (PIV 1-3), adenoviruses, rhinoviruses, coronaviruses, human bocavirus $(\mathrm{HBoV})$, enteroviruses and human metapneumovirus (hMPV) [4]. Despite the extensive laboratory detection and characterization of these viruses, almost $50 \%$ of ARTIs in children remain without an aetiological diagnosis [5].

hMPV of the family Paramyxoviridae was first identified in Netherlands in 2001 from nasopharyngeal aspirates (NPA) collected over a 20 years period [6]. The retrospective studies, however, indicate the presence of antibodies against hMPV in serum samples as old as 50 years. These findings support the notion that hMPV is not a new respiratory virus [5]. hMPV detection rates among children with ARTI are usually between 6 and 40\% [5]. hMPV has a global incidence of 5 to $10 \%$ in children with ARTIs especially causing infection in less than 2 year olds $[1,5]$. Seroprevelence studies indicate that more than $90 \%$ of children between the ages of 5 to 10 have already encountered the virus [1].

The clinical presentation of hMPV infection in children is indistinguishable from that of RSV infection [7]. hMPV causes clinical symptoms ranging from mild upper respiratory tract infection (URTI) to severe lower respiratory tract infection (LRTI). The most common symptoms of 
severe hMPV infection include bronchiolitis and pneumonia, which usually results in hospitalization [1]. In addition, other respiratory symptoms like cough, coryza, dyspnoea, fever and hypoxia may or may not be present in children with hMPV associated ARTI. In asthma patients, hMPV infection is known to cause exacerbation of asthma [8].

hMPV co-infects predominantly with RSV [5] although co-infections with PIV, $\mathrm{hBoV}$, adenoviruses, rhinoviruses, coronaviruses, enteroviruses and bacterial respiratory pathogens have also been reported [1]. Studies show that co-infection rates of hMPV lie within 5 to $17 \%$ [5]. Many studies reveal that there is no direct correlations between co-infection and disease severity [5]. Since viral ARTI being the second leading cause of hospitalization in developing countries in the South Asia including Sri Lanka, research on viral ARTI is a top priority. Although hMPV has been known as a cause of ARTI in children more than a decade, only a couple of published papers are available in Sri Lanka [9, 10]. Thus this study aimed to detect respiratory viruses including hMPV in a selected group of children affected by a mini outbreak of SARI presented to the Teaching Hospital, Peradeniya (THP), Sri Lanka.

From July to October 2014, twenty one children (1 to 4 years of age) with one or more ARTI symptoms were admitted to the THP. Demographic and Clinical data were collected using a questionnaire by interviewing respective guardians or parents of the child. NPA were collected from all 21 children with SARI by the paediatrician and transported to the Virology Laboratory of the Faculty of Medicine, University of Peradeniya immediately and each NPA sample was aliquoted into two different collection tubes and labeled for IFA and RT-PCR.

An indirect IFA was performed using an immunoflurescence assay (D3 UltraTM $^{\circledR}$, USA) for both screening and typing of eight different respiratory viruses (hMPV, influenza A and B, RSV, PIV 1-3 and adenovirus) [11]. Stained slides were examined under the fluorescence microscope and positive results were recorded based on the apple green fluorescence emission from infected cells. Viral RNA was extracted from $200 \mu \mathrm{L}$ of aliquoted NPA using QIAmp Viral RNA mini kit (Qiagen, Hilden, Germany). RNA extracts were subjected to a reverse transcriptase-polymerase chain reaction (RT-PCR) for the detection of hMPV using the Qiagen One Step RT-PCR kit (Qiagen, Hilden, Germany) targeting the $\mathrm{L}$ gene (5'CACCCCAGTCTTTCTTGAAA- $3^{\prime}$ ) of the viral genome. A thermal profile of reverse transcription at $50{ }^{\circ} \mathrm{C}$ for $30 \mathrm{~min}$, polymerase activation at $95{ }^{\circ} \mathrm{C}$ for $15 \mathrm{~min}, 40$ amplification cycles of denaturation at $94{ }^{\circ} \mathrm{C}$ for $1 \mathrm{~min}$, annealing at $55{ }^{\circ} \mathrm{C}$ for $1 \mathrm{~min}$ and polymerization at $72{ }^{\circ} \mathrm{C}$ for $1 \mathrm{~min}$ were used for the amplification. Finally Amplification products were separated by electrophoresis on a
$2.0 \%(\mathrm{w} / \mathrm{v})$ agarose gel and the $171 \mathrm{bp}$ target band was visualized following ethidium bromide staining (Fig. 1).

Of the 21 samples tested by IFA typing, 5 were positive, including one (4.8\%) each for hMPV and influenza A, 2 $(9.5 \%)$ were positive for RSV and $1(4.8 \%)$ was co-infected with adenovirus and PIV-2. Of the 21 samples tested via RT-PCR for hMPV, 18 (85.7\%) were positive. Of the 18 hMPV positive children, 2 were co-infected with RSV and one with influenza $\mathrm{A}$ with an overall co-infection rate of $16 \%$. Of the $18 \mathrm{hMPV}$ infected children, fourteen were males $(77.77 \%)$ with a male to female ratio of 3.5:1. The mean age of the hMPV infected children was 17 months (6-48 months).

All children with hMPV infection presented with cough, $90 \%$ of them had fever, apart from these symptoms, one child had sepsis and 3 had conjunctivitis. The clinical characteristics of the children positive for hMPV infection are shown in Fig. 2.

Of the $18 \mathrm{hMPV}$ infected children, 17 were breastfed at the time of the recruitment for the study, at least 4 were exposed to a smoker in the household and 11 of them grew up with at least 1 to 3 siblings. Majority of the hMPV infected children were clinically diagnosed for LRTI $(38.9 \%)$ and wheezing (33.3\%), the clinical diagnosis of hMPV infected children are shown in Fig. 3.

The WHO ranks ARTI as the second leading cause of death worldwide for children less than 5 years of age. The findings of the current study showed $85.7 \%$ (18/21) positivity for hMPV and this is considerably higher than the positivity rates reported by previous studies in which the positivity ranged from 6 to $40 \%$ in hospitalized children $[7,12,13]$. Recruiting children who were critically ill and hospitalized with severe acute respiratory symptoms for the study might have increased the detection of hMPV. As shown by many studies $[6,7,15,16]$, our findings also shows a male predominance in hMPV infection.

Our study indicates a $16 \%$ of co-infection of hMPV with other respiratory viruses. This agrees with many other studies that indicate co-infection rates within the range of 5

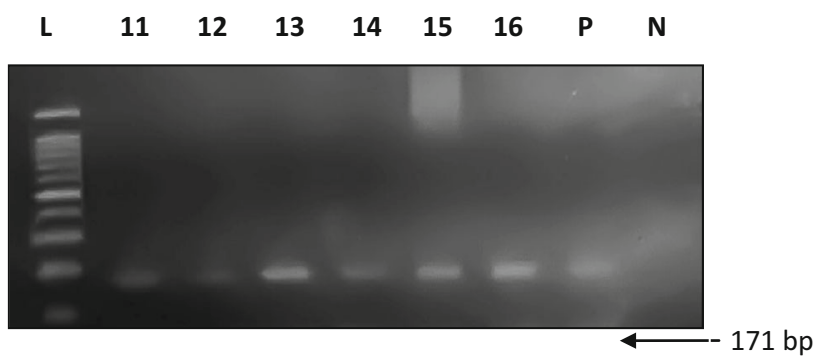

Fig. 1 hMPV detection by RT-PCR using the viral RNA extracts of NPA of children with severe respiratory symptoms. Lane L; $100 \mathrm{bp}$ ladder, Lanes 11-21; hMPV positive children's NPA samples, P; positive control, N; negative control 


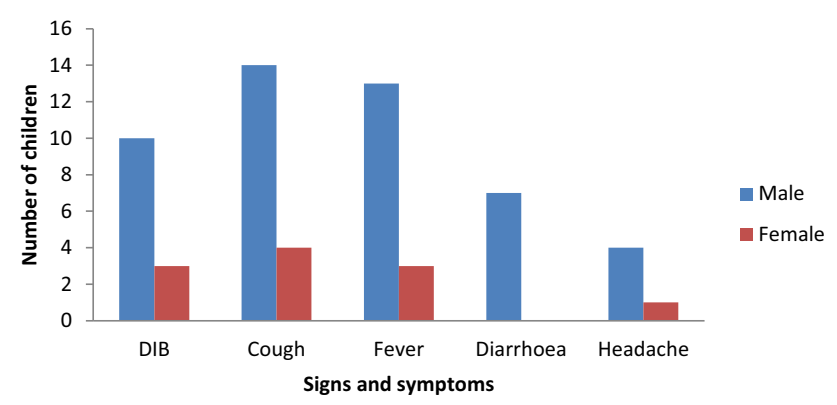

Fig. 2 Clinical characteristics of eighteen children tested positive for hMPV by RT-PCR. DIB - difficulty in breathing

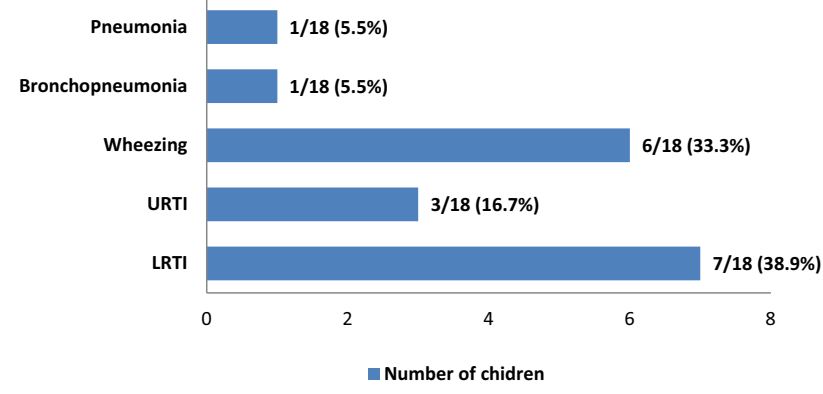

Fig. 3 Clinical diagnosis of hMPV infected children in the study sample. URTI-upper respiratory tract infection; LRTI-lower respiratory tract infection

to $17 \%$ [5]. Majority of the hMPV co-infections occurs with RSV [7] and this might be due to a mathematical chance for the presence of RSV as it is the number one cause of ARTI in children globally. In the current study, two out of the three co-infections detected were with RSV. Our study did not find a significant difference in terms of disease severity between hMPV co-infections and monoinfections as reported previously [5].

In this study, 2 children were diagnosed with pneumonia and 2 with bronchopneumonia and these findings are in agreement with other studies that symptoms range from mild URTI to severe LRTI [14]. In contrast, our study did not encounter a single case of bronchiolitis. Wheezing has been reported in hMPV infection in children $[1,14]$ and in our study wheezing was detected in 6 out of the 18 children. Fever, cough, and dyspnoea were common symptoms present in the current study sample with hMPV infection as reported by previous studies $[1,14]$. Seven of the 18 children had diarrhoea, although diarrhoea has been indicated in studies before in association with adenovirus infections, its association with hMPV infection has not been reported $[7,8]$.

The major limitation in the current study would be the small sample size $(n=21)$ and the short study period. Sequencing of the hMPV isolates from the current samples would have provided valuable information on the predominant and circulating genotypes of the virus in the study area. Nevertheless, findings of our study indicate the presence of hMPV associated SARI in children at least in the study area of Sri Lanka. Thus this study provides a platform for testing for hMPV in children with SARI in Sri Lanka.

Funding Grant No. RG/AF/2013/38/M from University of Peradeniya.

\section{Compliance with ethical standards}

Conflict of interest The authors declare that they have no conflict of interest.

Ethics approval and consent to participation The study was approved by the Ethical Review Committee of the Faculty of Medicine, University of Peradeniya, Sri Lanka. An informed written consent was obtained from the parents/guardians of the study participants.

\section{References}

1. Panda S, Mohakud NK, Pena L, Kumar S. Human metapneumovirus: review of an important respiratory pathogen. Int J Infect Dis. 2014;25:45-52.

2. GBD 2015 LRI Collaborators. Estimates of the global, regional, and national morbidity, mortality, and aetiologies of lower respiratory tract infections in 195 countries: a systematic analysis for the Global Burden of Disease Study 2015. Lancet Infect Dis. 2017; 17:1133-61.

3. Williams BG, Gouws E, Boschi-Pinto C, Bryce J, Dye C. Estimates of world-wide distribution of child deaths from acute respiratory infections. Lancet Infect Dis. 2002;2:25-32.

4. Tregoning JS, Schwarze J. Respiratory viral infections in infants: causes, clinical symptoms, virology, and immunology. Clin Microbiol Rev. 2010;23:74-98.

5. Williams JV. Human metapneumovirus: an important cause of respiratory disease in children and adults. Curr Infect Dis Rep. 2005;7:204-10.

6. Mullins JA, Erdman DD, Weinberg GA, Edwards KM, Hall CB, Walker FJ, et al. Human metapneumovirus infection among children hospitalized with acute respiratory illness. Emerg Infect Dis. 2004;10:700-5.

7. Peiris JSM, Tang WH, Chan KH, Khong PL, Guan Y, Lau YL, et al. Children with respiratory disease associated with metapneumovirus in Hong Kong. Emerg Infect Dis. 2003;9:628-33.

8. Schuster JE, Williams JV. Human metapneumovirus. Pediatr Rev. 2013;34:558-65.

9. Jayaweera JAAS, Noordeen F, Morel AJ, Pitchai FNN, Kothalawala S, Abeykoon AMSB, et al. Viral burden in acute respiratory tract infections in hospitalized children in wet and dry zones of Sri Lanka. Int J Infect Dis. 2016;45s:463.

10. Jayaweera JAAS, Noordeen F, Kothalaweala S, Pitchai FNN, Rayes MLM. A case series on common cold to severe bronchiolitis and pneumonia in children following human metapneumovirus infection in Sri Lanka. BMC Res Notes. 2018;11:127.

11. Muthulingam A, Noordeen F, Morel AJ. Viral etiology in hospitalized children with acute respiratory tract infection in the Kegalle area of Sri Lanka. J Pediatr Infect Dis. 2014;9:167-70.

12. Chung JY, Han TH, Kim BE, Kim CK, Kim SW, Hwang ES. Human metapneumovirus infection in hospitalized children with 
acute respiratory disease in Korea. $J$ Korean Med Sci. 2006;21:838-42.

13. Moe N, Stenseng IH, Krokstad S, Christensen A, Skanke LH, Risnes KR, et al. The burden of human metapneumovirus and respiratory syncytial virus infections in hospitalized Norwegian children. J Infect Dis. 2017;216:110-6.

14. Kahn JS. Epidemiology of human metapneumovirus. Clin Microbiol Rev. 2006;19:546-57.

15. Davis CR, Stockmann C, Pavia AT, Byington CL, Blaschke AJ, Hersh $\mathrm{AL}$, et al. Incidence, morbidity and costs of human metapneumovirus infection in hospitalized children. J Pediatr Infect Dis. 2016;5:303-11.

16. Schuster JE, Bulos NK, Faouri S, Shehabi A, Johnson M, Wang $\mathrm{L}$, et al. Human metapneumovirus infection in Jordanian children: epidemiology and risk factors for severe disease. Pediatr Infect Dis J. 2015;34:1335-41.

Publisher's Note Springer Nature remains neutral with regard to jurisdictional claims in published maps and institutional affiliations. 